\title{
A RELAÇÃO DE PROBLEMAS NO PROCESSAMENTO DA LINGUAGEM COM DIFICULDADES DE APRENDIZAGEM
}

\author{
Danielli Cristina de Lima Silva ${ }^{1}$ \\ Eduardo Lucas de Sousa Eneas ${ }^{2}$ \\ José Ferrari Neto ${ }^{3}$ \\ Henrique Miguel de Lima ${ }^{4}$
}

Resumo. O presente trabalho surge da necessidade de fazer um resgate da discussão de um artigo publicado na VII Conferência Internacional de Linguística e Cognição que ocorreu em 2015 no Campus I da Universidade Federal da Paraíba - UFPB com anais publicados. As dificuldades de aprendizagem denunciam que algo não anda bem no processo de aprendizagem, sabendo disso, há inúmeros problemas relacionados a esses problemas dentre eles: metodologias de ensino tradicionais, percepção visual e principalmente as dificuldades no processamento da linguagem. Essas dificuldades abrangem os fatores cognitivos e sociais, e caso não sejam prevenidas/tratadas com pontualidade, levando em consideração que a aprendizagem é cumulativa, podemos ter comprometimentos em relação ao desempenho escolar. A principal finalidade deste artigo é retomar os dados apresentados da revisão sistemática intitulada de Estudo sobre a relação de dificuldades no processamento da linguagem com problemas escolares, a qual foi apresentado a análise de 20 artigos em bancos de dados Scielo e Pepsic com o descrito "processamento + linguagem", foram selecionados artigos de 2010 a 2014, publicados nas línguas inglesas, portuguesa e espanhola que investigaram o desempenho de crianças e adolescentes. Os resultados apontados demonstraram que escolares com dificuldade no processamento da linguagem apresentam baixo rendimento em leitura e escrita, pelo fato de demandarem a utilização da memória de trabalho e conhecimento lexical para essas atividades. A compreensão destes fatores podem contribuir para professores, psicopedagogos e familiares a lidarem com essas dificuldades e consequentemente contribuir para sanar as dificuldades de aprendizagem.

Palavras-chave: Processamento da linguagem. Dificuldade de Aprendizagem. Ensino.

\section{THE RELATION OF LANGUAGE PROCESSING PROBLEMS WITH LEARNING DIFFICULTIES}

\footnotetext{
Abstract. The present work arises from the need to make a rescue of the discussion of an article published in the VII International Conference on Linguistics and Cognition

${ }^{1}$ Universidade Federal da Paraíba - (UFPB) João Pessoa - PB - Brasil - limaanacrisdani@ gmail.com

${ }^{2}$ Universidade Federal da Paraíba - (UFPB) João Pessoa - PB - Brasil - eduardolucas.vip@ hotmail.com

${ }^{3}$ Universidade Federal da Paraíba - (UFPB) João Pessoa - PB - Brasil Coordenador do Programa de PósGraduação em Linguística - PROLING/UFPB

${ }^{4}$ Universidade Federal da Paraíba - (UFPB) João Pessoa - PB - Brasil, Universidade Federal de Campina Grande - UFCG
} 
that took place in 2015 in Campus I of the Federal University of Paraíba - UFPB with published annals. Learning disabilities report that something is not right in the learning process, knowing that there are innumerable problems related to these problems: traditional teaching methodologies, visual perception and especially difficulties in language processing. These difficulties include cognitive and social factors, and if they are not prevented / treated with punctuality, taking into account that learning is cumulative, we can have commitments regarding school performance. The main purpose of this article is to return the data presented in the systematic review entitled Study on the relationship of difficulties in the processing of language with school problems, which was presented the analysis of 20 articles in Scielo and Pepsic databases with the described "processing + language, "articles were selected from 2010 to 2014, published in the English, Portuguese and Spanish languages that investigated the performance of children and adolescents. The results pointed out that students with difficulty in language processing have low reading and writing performance due to the fact that they require the use of working memory and lexical knowledge for these activities. The understanding of these factors can contribute to teachers, psychopedagogues and family members to deal with these difficulties and consequently help to solve learning difficulties.

Keywords: Language processing. Learning Difficulty. Teaching.

\section{Introdução}

O presente trabalho surge da necessidade de dar seguimento as discussões quanto a relação de dificuldades de aprendizagem com problemas no processamento da linguagem a partir da revisão sistemática do artigo Estudo sobre a relação de dificuldades no processamento da linguagem com problemas escolares, além da pertinência de ampliar o debate com uma discussão sobre ensino, linguística cognitiva e neurociência de forma mais pontual.

As dificuldades de aprendizagem podem atingir pessoas de qualquer faixa etária, no entanto, a maior preocupação acaba sendo com crianças e adolescentes, uma vez que esse público em termos de desenvolvimento passam por etapas educacionais das mais simples as mais complexas em um período cuja esimulação e superação de qualquer dificuldade são capazes de repercutir ao longo de toda a vida escolar e profissional.

Vale ressaltar que a proposta de discussão de dificuldades de aprendizagem é de maneira geral e, portanto, pode ser algo que acometa até mesmo pessoas que não possuem nenhum disturbio de aprendizagem e/ou psicopatologia. $\mathrm{O}$ mesmo se aplica

RPI Revista de Pesquisa Interdisciplinar, Cajazeiras, v. 2, n. 2, 149-156, jun/dez. de 2017. 
aos problemas de processamento, conforme Silva, Eneas e Ferrari-Neto (2016, p. 02): “O processamento da linguagem em estudo para ser relacionado com dificuldades de aprendizagem precisa ser analisado tanto em pessoas com desenvolvimento da linguagem típico quanto os que possuem Distúrbio Específico de Linguagem (DEL)”. Podemos perceber que dependendo da frequência com que estes problemas no processamento em situações cotidianas ou escolares, a intervenção dos educadores torna-se necessária.

Mediante a importância da temática a retomada desses dados e a ampliação das análises na tentativa de um debate mais próximo do âmbito educacional para promover melhorias nas práticas de ensino e um ambiente param se repensar as possíveis causas quando diante das dificuldades de aprendizagem. Smith e Strick (2012) apontam os problemas de processamento da linguagem como prováveis fatores que implicam nas dificuldades de aprendizagem, tendo em vista que estes problemas de linguagem atrapalham o funcionamento do cérebro.

Para seguirmos a discussão da relação dos problemas de processamento da linguagem com dificuldades de aprendizagem utilizamos dados de um estudo anterior, CARROL (2004), OLIVEIRA e. al. (2011), SMITH E STRICK (2012), dentre outros que nos auxiliam a compreender com mais clareza a complexa relação desses fenômenos e possíveis implicações na aprendizagem.

Diante do exposto, o presente artigo tem por objetivo geral fazer uma discussão a partir da retomada de um trabalho indicando a relação de dificuldades no processamento da linguagem com problemas escolares com intuito de aproximar o debate linguístico do âmbito educacional. E, mais especificamente: a) Discutir a relação dos problemas de processamento da linguagem com dificuldades de aprendizagem; b) Realizar uma breve apresentação sobre o processamento da linguagem e as dificuldades de aprendizagem, e por fim, c) demonstrar a importância do conhecimento da relação de dificuldades de aprendizagem com problemas de processamento da linguagem e a possíveis implicações no âmbito escolar.

\section{Dificuldades de Aprendizagem}

A aprendizagem é uma preocupação constante de pais e professores, por saberem da importância que o aprender tem para o desenvolvimento de maneira geral. 
Trata-se de "[...] processos complexos dos quais recorremos a explicações biopsicossociais para compreendê-la em sua totalidade." (SILVA; ENEAS; FERRARINETO, 2016, p. 03).

Nesse processo de aquisição de novos saberes podem ocorrer as chamadas dificuldades de aprendizagem, estas se caracterizam por problemas que atrapalham o “[...] processamento adequado das informações.” (SMITH e STRICK, 2012, p. 38). As dificuldades de aprendizagem podem se manifestar basicamente no processamento da linguagem, na coordenação motora fina, atenção e percepção visual; elas são responsáveis por problemas no desempenho escolar e acadêmico, a qual envolve inúmeros aspectos de modo a não ser atribuído unicamente a um problema específico, mas uma série de fatores que combinados tornam mais difícil a aprendizagem plena.

Ainda a respeito das dificuldades de aprendizagem:

[...] É complicado identificar uma dificuldade de aprendizagem, pois a gravidade da situação varia consideravelmente de pessoa para pessoa e pode acontecer em indivíduos que possuam algum distúrbio/psicopatologia ou não. (SILVA; ENEAS, FERRARI-NETO, 2016, p. 03)

Diane dessa afirmação fica evidente a importância da atenção por parte dos educadores, pais e profissionais das áreas de linguagem, além de psicopedagogos sobre esses aspectos concernentes a dificuldade de aprendizagem, tendo em vista a necessidade de fornecer apoio aos indivíduos com dificuldades de aprendizagem.

Existem comportamentos que os profissionais da educação e pais devem estar atentos para identificar, muitos consideram como preguiça ou desinteresse, tais atribuições a estes indivíduos pode piorar o quadro, Por isto vale observar, segundo Silva; Eneas e Ferrari-Neto (2016, p. 04):

- Dificuldade para seguir instrução no qual é necessário muitas repetições para não haver engano;

- $\quad$ Imaturidade social em função de um comportamento inferior a idade cronológica;

- Dificuldade para a conversação nesse caso fala em demasia ou não encontra as palavras;

- Inflexibilidade realiza as atividades sempre de uma mesma forma e recusa ajuda;

- $\quad$ Planejamento e habilidades organizacionais a noção de tempo e capacidade de resolver várias tarefas são comprometidas;

- Distração perde objetos apresenta esquecimento de situações cotidianas e responsabilidades agendadas;

- $\quad$ Falta de destreza parece não ter coordenação;

- $\quad$ Falta de controle dos impulsos dificuldades em esperar a vez de falar, expõe suas observações sem nenhuma ponderação;

Com isto, podemos considerar o quanto as dificuldades de aprendizagem podem ser percebidas no ambiente doméstico e no ambiente escolar. Fica evidente a relevância 
do diálogo entre família e escola para o acompanhamento das rotinas de aprendizagem das crianças e adolescentes.

\title{
Possíveis relações entre processamento da linguagem e dificuldades de aprendizagem
}

A linguagem tem papel fundamental no desenvolvimento e na possibilidade de os indivíduos tornarem os próprios conhecimentos mais complexos em função de, nas interações e nos estímulos diários, utilizar da língua(gem) para acessar o mundo ao redor. É através do processamento da linguagem que o indivíduo pode interpretar o mundo. Segundo Camara Jr. (2007, p. 15)

\begin{abstract}
A linguagem é algo trivial em nossa vida social. (...). Embora não seja linguagem um fenômeno biológico como o caminhar; mas uma criação social baseada nas capacitações biológicas, o falar torna-se tão mecânico na vida que é considerado autoevidente e não se faz qualquer esforço para analisá-lo.
\end{abstract}

Os indivíduos para interpretar o mundo ao redor precisam processar os fatos ocorridos através do estímulo, este por sua vez tem início com o processamento fônico, ou seja, o processamento do som. Para Carrol (2004) o processamento fônico ocorre em três níveis, conforme quadro elaborado para melhor entendimento a partir de (SILVA; ENEAS; FERRARI-NETO, 2016, p. 07):

\begin{tabular}{|l|l|l|}
\hline \multicolumn{1}{|c|}{ Nível Fonético } & \multicolumn{1}{c|}{ Nível Fonológico } & \multicolumn{1}{c|}{ Nível prosódico } \\
\hline $\begin{array}{l}\text { Sons em suas propriedades } \\
\text { puras }\end{array}$ & $\begin{array}{l}\text { Categorização dos fonemas } \\
\text { e reconhecimento de seus } \\
\text { valores }\end{array}$ & $\begin{array}{l}\text { Decifração de propriedades } \\
\text { acústicas e dos respectivos } \\
\text { valores. }\end{array}$ \\
\hline
\end{tabular}

Estas primeiras etapas são essenciais para o avanço da linguagem desses indivíduos, por isso a necessidade do conhecimento a respeito para evitar intervenções tardias. De acordo com, Silva; Enéas e Ferrari-Neto, 2016, p. 08):

O processamento da linguagem não delimita - se ao processamento fônico, porém, trata-se do caminho para o desenvolvimento da leitura e escrita que serão fundamentais para um bom desempenho escolar, uma vez que é por meio destas fixações de parâmetros da língua nativa que o falante consegue realizar a leitura e escrita de vocábulos da língua materna. 
Diante disso, as considerações sobre os problemas de processamento da linguagem pesarem no desempenho dos indivíduos, ganham significado no cenário de investigação dos fenômenos associados a questões mais pontuais de aprendizagem. Segundo Smith e Strick (2012, p. 51): “As crianças com dificuldades de processamento da linguagem podem ser lentas na aprendizagem da fala e podem usar sentenças mais curtas, vocabulários menores e uma gramática mais pobre do que seus irmãos em uma idade similar." A atenção para estas informações servem para obter indícios de possíveis dificuldades de aprendizagem, no entanto não devemos tomar estes fatos como determinísticos.

Segundo Befi-lopes (2010) as crianças com desenvolvimento normal da linguagem (DNL) em comparação com as crianças com distúrbios específicos da linguagem (DEL) não possuem problemas nas representações fonológicas, ao contrário das crianças com DEL. Podemos presumir, a partir disso, que na fala há uma melhor compreensão quando se opta por uma linguagem mais simples.

As dificuldades de aprendizagem ficam mais evidentes em relação ao processamento da linguagem quando as crianças em contato com formas de linguagem mais complexas e não conseguem obter sucesso no processamento da linguagem. Segundo Smith e Strick (2012, p. 53): “Os problemas com a compreensão tendem a piorar quando a linguagem que está sendo usada é complexa: uma criança que consegue lidar sem dificuldades com uma ordem." Muita das vezes quando isso ocorre os pais/professores não entendem que a fala utilizada pode ter dificultado a compreensão.

Há inúmeras situações além dessa citada acima que demonstram a relação entre problemas no processamento da linguagem e dificuldades de aprendizagem, tais como o de Frota (2010) crianças com este tipo de problema também passa a ter dificuldade de leitura/escrita, nesse sentido Gavyha (2010) aponta a necessidade de intervenção múltipla em indivíduos para que consigam superar essas dificuldades.

Os estudos realizados por diversos estudiosos, além dos citados acima, apresentam resultados que nos levam a confirmar esta relação, pois os que participantes com falhas/problemas no processamento da linguagem independente de possuírem algum distúrbio específico ou não (SILVA; ENEAS, FERRARI-NETO, 2016), necessitam de intervenção para melhorar as habilidades linguísticas e superar as dificuldades (BRETANHA, 2012). No tocante a pessoas com distúrbios específicos de 
linguagem, o ideal é trabalhar a partir de método fônico (OLIVEIRA et. al., 2011), ou seja, com intervenções baseadas no estímulo auditivo e significação de símbolos gráficos.

Desta forma, os problemas de processamento da linguagem precisam estar nas pautas de investigação ao se detectar uma dificuldade de aprendizagem em crianças, adolescentes e até mesmo adultos, por sabermos que independente da fase de desenvolvimento as pessoas são expostas a situações que requerem a aquisição de novos conhecimentos.

\section{Importância do conhecimento sobre as relações de processamento da linguagem e dificuldade de aprendizagem para a educação}

A educação trata-se de uma das formas pelas quais os indivíduos tem acesso a hábitos, costumes, comportamentos responsáveis pela socialização bem como a integração de conhecimentos de várias áreas que reunidas consolidam as aprendizagens necessárias, é pela aprendizagem que o indivíduo consegue permanecer em evolução.

Com isto, podemos ter uma noção mais precisa do quanto as dificuldades de aprendizagem comprometem o desenvolvimento e por pensar assim, os educadores e pais se preocupam quando se deparam com o esforço dos alunos/filhos na tentativa de achar soluções para estes quadros.

A respeito das dificuldades de aprendizagem e problemas no processamento quando relacionado é preciso ter noção de que, os com problemas específicos de linguagem ou algum distúrbio, precisam de intervenções desde muito cedo para evitar a persistência desses problemas de aprendizagem. Quanto as habilidades linguísticas que permitem avanços no desempenho escolar:

\footnotetext{
Em termos de aprendizagem ter problemas nessas habilidades [...] tem impacto no desempenho escolar, uma vez que o conhecimento formal requer uma série de habilidades linguísticas que subjazem a cognição dos indivíduos em processo desenvolvimento normal ou atípico dos quais a rotina de exigências formais para o aprendizado é constante e ao considerar os níveis hierárquicos dos processos mentais para a leitura, do processamento fônico ao semântico, é preciso fazer o acompanhamento desses indivíduos para evitar problemas que acarretem uma eminente dificuldade de aprendizagem. (SILVA; ENEAS; FERRARI-NEO, 2016, p. 18).
} 
Para a aprendizagem plena dos alunos é importante a parceria entre os educadores de tal maneira que seja possível fornecer o apoio para a efetivação de novos conhecimentos, sem desrespeitar as etapas do desenvolvimento nem os mecanismos cerebrais envolvidos neste processo. $\mathrm{O}$ intuito dessas discussões além de demonstrar o quanto é complexo o processo de aprendizagem é expor a ausência de culpados diante de uma dificuldade de aprendizagem que há algo mais relevante, a possibilidade de encontrar estratégias de auxílio para pessoas com dificuldade de aprendizagem.

\section{Considerações Finais}

Neste artigo objetivamos trazer de forma simples e convidativa sobre a relação dos problemas de aprendizagem com dificuldades de aprendizagem, com a finalidade de estabelecer um elo convidativo para o leitor diante da necessidade de um debate mais aprofundado a respeito desse assunto, bem como ampliar a proximidade com estudiosos que se interessam pela temática abordada.

As dificuldades de aprendizagem podem acontecer por inúmeros fatores, $\mathrm{n}$ entanto quando estão ligados a problemas no processamento da linguagem os comprometimentos são por demais severos. Tal consideração requer medidas para reverter quadros de dificuldades, estes só são possíveis com o empenho de pais e educadores, bem como a busca por auxílio de outros profissionais para elaborar intervenções pontuais e capazes de possibilitar melhorias nas habilidades linguísticas e consequentemente no desempenho escolar.

Para os profissionais da educação ter conhecimento dessas implicações relativas aos possíveis problemas que causam as dificuldades de aprendizagem torna - os mais conscientes na hora de agir nas situações cotidianas de aprendizagem. Além disso, er a percepção de que muitas das vezes um comportamento do qual é ido como de criança preguiçosa pode ser um indício da confusão mental que as dificuldades de aprendizagem podem causar em razão do problema de processar uma informação linguística. 


\section{Referências}

CASTELLS, Manuel. Paraísos Comunais: identidade e significado na sociedade em rede. In. . O poder da identidade. (A era da informação: economia, sociedade e cultura).Rio de Janeiro: Paz e Terra, 2002.

COELHO, Wilma Baía. A cor ausente:um estudo sobre a presença do negro na formação de professores- Pará, 1970-1989. Belo Horizonte: Editora Unama, 2006.

GOMEZ, Margarita Victoria. Educação em rede: uma visão emancipadora. São Paulo: Cortez: Instituto Paulo Freire (Guia da escola cidadã; v.11), 2004.

LÉVY, Pierre. AsTecnologias da Inteligência: o futuro do pensamento na era da informática. Rio de Janeiro: Editora 34, 1993.

PALLOFF, Rena M.; PRATT, Keith. O aluno virtual: um guia para trabalhar com estudantes on-line Porto Alegre: Artmed, 2004.

Construindo comunidades de aprendizagem no ciberespaço estratégias eficientes para as salas de aula on-line. Porto Alegre: Artmed, 2002.

PERRENOUD, Philippe. A prática reflexiva no ofício de professor: profissionalização e razão pedagógica. Porto Alegre: Artmed, 2002.

RIBEIRO, Otacílio José. Educação e novas tecnologias: um olhar para além da técnica. In: COSCARELLI, Carla Viana. RIBEIRO, Ana Elisa (orgs). Letramento Digital aspectos sociais e possibilidades pedagógicas. Belo Horizonte: Ceale; Autêntica, 2005.

TAKEUCHI, Hirotaka; NONAKA, Ikujiro. Gestão do conhecimento. Tradução: Ana Thorell. Porto Alegre: Bookman, 2008. 